\title{
Ultrafast charge transfer dynamics in 2D Covalent Organic Frameworks/Re-complex hybrid photocatalyst for $\mathrm{CO} 2$ reduction: hot electrons vs. cold electrons
}

\author{
Qinying Pan \\ Technical University of Denmark \\ Mohamed Abdellah \\ Lund University \\ Yuehan Cao \\ Southwest Petroleum University https://orcid.org/0000-0003-4073-6162 \\ Yang Liu \\ Southwest Petroleum University \\ Weihua Lin \\ Lund University \\ Jie Meng \\ Technical University of Denmark https://orcid.org/0000-0002-3813-5221 \\ Quan Zhou
}

Technical University of Denmark

Zonglong Li

Tsinghua University

Hao Cui

Tsinghua University

David Tanner

TU Denmark

Mahmoud Abdel-Hafiez

Uppsala University

Ying Zhou

Southwest Petroleum University https://orcid.org/0000-0001-9995-0652

Tonu Pullerits

Lund University https://orcid.org/0000-0003-1428-5564

Sophie Canton

European XFEL https://orcid.org/0000-0003-4337-8129

Hong Xu

Tsinghua University https://orcid.org/0000-0001-7918-1454 
Kaibo Zheng ( $\sim$ kzheng@kemi.dtu.dk)

Technical University of Denmark

\section{Article}

Keywords: Sensitizers, Excited State Dynamics, Time-dependent DFT Calculations, Backward Recombination, Long-lived Free Electrons

Posted Date: May 3rd, 2021

DOl: https://doi.org/10.21203/rs.3.rs-448398/v1

License: (c) (i) This work is licensed under a Creative Commons Attribution 4.0 International License. Read Full License

Version of Record: A version of this preprint was published at Nature Communications on February 11th, 2022. See the published version at https://doi.org/10.1038/s41467-022-28409-2. 


\section{Abstract}

Rhenium(I)-carbonyl-diimine complexes are promising photocatalysts for $\mathrm{CO}_{2}$ reduction. Covalent organic frameworks (COFs) can be perfect sensitizers to enhance the reduction activities. Here we investigated the excited state dynamics of COF (TpBpy) with 2,2'-bipyridine incorporating $\operatorname{Re}(\mathrm{CO}){ }_{5} \mathrm{Cl}(\operatorname{Re}-\mathrm{TpBpy})$ to rationalize the underlying mechanism. The time-dependent DFT calculation first clarified excited state structure of the hybrid catalyst. The studies from transient visible and infrared spectroscopies revealed the excitation energy-dependent photo-induced charge transfer pathways in Re-TpBpy. Under low energy excitation, the electrons at the LUMO level are quickly injected from Bpy into Re' center (1-2 ps) followed by backward recombination (13 ps). Under high energy excitation, the hot-electrons are first injected into the higher unoccupied level of $\mathrm{Re}^{\mathrm{l}}$ center (1-2 ps) and then slowly relax back to the HOMO in COF (24 ps). There also remains long-lived free electrons in the COF moiety. This explained the excitation energydependent $\mathrm{CO}_{2}$ reduction performance in our system.

\section{Introduction}

The solar-driven photocatalytic conversion is regarded as one of the most promising approaches for $\mathrm{CO}_{2}$ transformation to tackle the rising issue of greenhouse gas emission ${ }^{1-7}$. Among the state-of-the-art photocatalysts for $\mathrm{CO}_{2}$ reduction, Rhenium(I)-carbonyl-diimine complexes have attracted considerable attention due to their high photocatalytic quantum yield and selectivity for $\mathrm{CO}_{2}$ reduction ${ }^{8-12}$. In general, one photon excitation in the molecule can usually trigger one-electron transfer highly endergonic for $\mathrm{CO}_{2}$ reduction ${ }^{10}$. The one-electron reduced (OER) Re-complexes is still energetically favorable to donate the second electron. This is vital for $\mathrm{CO}_{2}$ reduction which is usually mediated by a two-electron transfer process $^{8,9,11-15}$. However, the short excited-state lifetime and accessible annihilation of multi-excitons in the molecules remains an obstacle for such a process. In addition, the intrinsic ${ }^{1} \mathrm{MLCT}$ absorption transition in Re-complexes covers a limited spectral region (350-450 nm $)^{13,16}$. Therefore Re-complexes are usually paired with suitable photosensitizer to provide efficient and long-lived excited electrons for photocatalytic reaction ${ }^{17-20}$. One emerging strategy is to immobilize such Re-complexes into porous scaffolds such as covalent organic frameworks (COFs) to construct heterogeneous molecular photocatalysts ${ }^{21-24}$. COFs are porous crystalline polymeric materials constructed by covalently bonded organic building blocks with highly ordered and periodic network structures ${ }^{25,26}$. The extended $\pi$ conjugation ensures broad light absorption and high charge conductivity ${ }^{22}$. Equally important, the porous structure with the large surface area provides numerous active sites for $\mathrm{CO}_{2}$ capture and catalytic reaction ${ }^{27,28}$. Recent studies reported high photocatalytic $\mathrm{CO}_{2}$ reduction activity on such Recomplex/COFs hybrid systems, which were rationalized by the efficient intramolecular charge transfer (ICT) from COF units to Re-complexes ${ }^{22,23}$ However, the detailed charge transfer dynamics have not been thoroughly understood. In fact, the charge transfer pathways in Re-complex/COFs hybrids can be complicated. Taking the two-dimensional (2D) donor-acceptor (2D D-A) COFs as an example, the high 
degree of $\pi$-conjugation imparts a semiconducting behavior while the polaron formation reduces the exciton binding energy ${ }^{29,30}$. In addition, the early-time (i.e. subpicosecond to picosecond) excited state dynamics in COFs comprises multi-steps inter-unit charge transfer. Such a process may compete or modulate the ICT, which should occur within a similar time scale ${ }^{29}$.

Another imperative topic worth investigating is the dynamics of the hot carriers excited by the high energy photons above the bandgap of the COFs. In traditional semiconductors, the hot carriers quickly thermalize to the band edge. Harvesting the energy of the hot carriers without losing it as thermal energy is a crucial step to break the $33 \%$ Shockley-Queisser thermodynamic efficiency limit of a standard singlejunction solar cell ${ }^{31}$. The same gain in energy conversion efficiency can be expected in photocatalytic reactions ${ }^{31-35}$. Considering existence of inter-unit charge transfer during the excited state relaxation process in 2D D-A COFs which can be governed by the molecular assembly, the hot carrier collection should be feasible in Re-complex/COFs but requires experimental evidence.

Herein, taking a 2D COF (TpBpy) with 2,2'-bipyridine incorporating $\operatorname{Re}(\mathrm{CO})_{5} \mathrm{Cl}$, named Re-TpBpy, as an example, we demonstrate the excited state dynamics and charge transfer process in the hybrid. The timedependent density functional theory (TD-DFT) calculations first displays the available electronic transition after excitation of the hybrids. The femtosecond transient visible (fs-TA) and Time-resolved infrared (fs-TRIR) absorption spectroscopies provide complementary information for the charge transfer dynamics. Photons with energy close to the bandgap of the COF directly excite the electron from ground state to the excited states in Bpy moiety followed by sub-picosecond electron injection to $\operatorname{Re}(\mathrm{CO})_{3} \mathrm{Cl}$. However, the injected electrons rapidly undergo geminated recombination with the residual holes in the COFs moiety within $13 \mathrm{ps}$. When the excitation is well above the absorption edge, the hot electrons and holes are initially generated evenly crosswise the entire COF. Hot electrons would directly inject into the higher energy orbital of $\operatorname{Re}(\mathrm{CO})_{3} \mathrm{Cl}$ within $2 \mathrm{ps}$ and rebound to the Bpy within $24 \mathrm{ps}$. The hot holes slowly relax to the HOMO level of COFs (340 ps). The prolonged excited electron lifetime in $\operatorname{Re}(\mathrm{CO})_{3} \mathrm{Cl}$ and the higher energy levels, together with the additional long-lived free electrons in COF moiety contributes as merits for a two-electron transfer mediated $\mathrm{CO}_{2}$ catalytic reaction. Our study rationalizes the excitation energy-dependent photocatalytic reaction mechanism in such Re-complex/COFs hybrid system, which can be beneficial for future materials engineering towards optimal photocatalytic performance.

\section{Results}

\section{Characterization of TpBpy and Re-TpBpy.}

Re-TpBpy is constructed by long-range ordered 2D sheets through the layer to layer stacking as shown in Fig.1a with the synthesis process summarized in Fig. S1. The characterization of TpBpy and Re-TpBpy was achieved by powder X-ray diffraction (PXRD), X-ray photoelectron spectroscopy (XPS), and Fourier Transform Infrared (FT-IR). PXRD patterns of both TpBpy and Re-TpBpy match well with the simulated AA stacking structure in the hexagonal space group (P6). After Re-complex incorporation, the crystalline 
structure of TpBpy remains unchanged. The XPS spectra confirmed the anchoring of Re-complex to the host TpBpy only through its bipyridinic units ${ }^{24}$ (for detailed XRD and XPS characterization, see Fig. S2 and S3 in the supporting information). The FT-IR spectrum (Fig. 1b) of Re-TpBpy manifest the preserved chemical functionalities of pristine TpBpy while two additional peaks arising at $2025 \mathrm{~cm}^{-1}$ and 1887 $\mathrm{cm}^{-1}$ can be attributed to the $\mathrm{C}=0$ stretching vibration in the $\mathrm{Re}(\mathrm{CO})_{3} \mathrm{Cl}$ moiety ${ }^{23}$. Additionally, compared with the FT-IR spectrum of $\operatorname{Re}(\mathrm{CO})_{5} \mathrm{Cl}$ and TpBpy, the $\mathrm{C}=\mathrm{O}$ stretching bonds and the broadened $\mathrm{C}-\mathrm{N}$ peak in the Re-TpBpy are slightly red-shifted, indicating the coordination of $\operatorname{Re}(\mathrm{CO})_{3} \mathrm{Cl}$ to the bipyridinic $\mathrm{N}$ atoms in the TpBpy. Our FT-IR result also excludes the existence of residual Tp and Bpy in both assynthesized TpBpy and Re-TpBpy.

\section{Steady-state spectroscopic study}

Steady-state absorption and PL spectra first clarified the ground state features of the Re-TpBpy hybrid and its individual units (Fig. 2a). The absorption spectra of the pure Tp and Bpy both showed a single distinct absorption band of Tp (350-400 nm) and Bpy $(380 \mathrm{~nm})$. The absorption spectrum of Re-Bpy is identical to Bpy except for a subtle blue shift (maxima absorption at $360 \mathrm{~nm}$ ) owing to the metal-toligand charge transfer (MLCT) $\left[d(R e)-\pi^{\star}(\text { bpy })\right]^{36,37}$ On the contrary, the absorption spectrum of TpBpy exhibits dual absorption bands with a narrow bipyridine $n-\pi^{*}$ transition band (324 nm) as well as a broad band at $508 \mathrm{~nm}$ for delocalized $\pi$ electrons ${ }^{38}$. The similar absorption spectra between Re-TpBpy and TpBpy suggest the high ligand stability (i.e. chromophore function) after functionalization ${ }^{24}$. The slight blue shift of the spectrum for Re-TpBpy should be due to the metal-to-ligand charge transfer (MLCT) $\left[\mathrm{d}(\mathrm{Re})-\pi^{\star}(\mathrm{bpy})\right]$.

Fig. 2a also shows the emission spectra of all samples excited at the band edge( $530 \mathrm{~nm}$, blue curve) and well above the band edge ( $400 \mathrm{~nm}$, red curve). Tp and Bpy share the same emission band at $500 \mathrm{~nm}$ upon the $400 \mathrm{~nm}$ excitation with moderate Stokes shift. In contrast, the emission spectrum of the Re-Bpy $(\lambda e m=585 \mathrm{~nm})$ is broad with large Stokes shift $(\Delta \lambda=225 \mathrm{~nm})$, which can be attributed to the existence of a triplet metal-to-ligand charge-transfer $\left({ }^{3} \mathrm{MLCT}\right)$ states ${ }^{39}$. When excited at $530 \mathrm{~nm}$, the emission spectra of TpBpy and Re-TpBpy are identical with emission bands at $620 \mathrm{~nm}$. This indicates similar emissive states from delocalized $\pi$ electrons in the two samples. When excited at higher energy $(400 \mathrm{~nm})$, the emission spectra of TpBpy and Re-TpBpy exhibit dual emission bands (i.e., $427 \mathrm{~nm}$ and $531 \mathrm{~nm}$ for TpBy, $431 \mathrm{~nm}$, and $562 \mathrm{~nm}$ for Re-TpBpy). The origins of such multi-emission bands in COFs can be complicated where one hypnosis is the radiative recombination in the single units of the $\mathrm{COF}^{40}$ We can obtain the same conclusion in the following analysis of the excited states. In summary, the optical transitions of TpBpy and Re-TpBpy are distinct from the ones of their original building block units. We can further calculate the optical band gaps $\left(E_{g}\right)$ of Tp, Bpy, Re-Bpy, TpBpy and Re-TpBpy from the Tauc plots of the absorption spectra (Fig. 2b) to be $3.14 \mathrm{eV}, 2.79 \mathrm{eV}, 2.90 \mathrm{eV}, 2.26 \mathrm{eV}$, and $2.17 \mathrm{eV}$, respectively. The characterization with XPS determines the valence band maximum (VBM) position of Tp, Bpy, Re-Bpy, 
TpBpyand Re-TpBpy to be $1.43 \mathrm{eV}, 1.99 \mathrm{eV}, 0.96 \mathrm{eV}, 1.89 \mathrm{eV}$, and $0.99 \mathrm{eV}$ corresponding to the Fermi level, respectively (Fig. 2c). Based on such values, we can determine the band energy alignment of the samples, as shown in Fig. $2 \mathrm{~d}$.

\section{Excited state structures caculation}

In order to obtain insight into the excited-state structure of the compounds, we used a triformylphloroglucinol (Tp) terminated bipyridine (Bpy) molecular fragment (Fig. 3) to represent the COF structure. The TD-DFT at the M06-L ${ }^{41-44} /$ def 2 TZVP ${ }^{45,46}$ level of theory has been employed to calculate the electronic structure and model the electronic transitions. Fig. 3 exhibits the calculated electronic excitation spectra of the TpBpy and Re-TpBpy (orange curves), which can resemble the experimental absorption spectra (red curves). The calculated spectrum of TpBpy mainly consists of two electronic excitation bands at $528 \mathrm{~nm}\left(\mathrm{~S}_{1}\right)$ and $436 \mathrm{~nm}\left(\mathrm{~S}_{2}\right)$, where high energy band $\mathrm{S}_{2}$ is equally contributed by the electronic transition (HOMO-3 $\rightarrow$ LUMO+1) and (HOMO $\rightarrow$ LUMO+2). The low energy band $S_{1}$ is dominated by the electronic transition from HOMO to LUMO level as illustrated in Fig. 3a. The low energy optical transition only occurs at the Bpy moiety whereas the high energy transition involves the electron population at both Tp and Bpy in the COF moieties. The modeled spectrum of Re-TpBpy also shows two pronounced electronic excitation bands where the high energy band is contributed by two electronic transition $\mathrm{S}_{3}\left(\mathrm{HOMO} \rightarrow\right.$ LUMO+2, $427 \mathrm{~nm}$ ) and $\mathrm{S}_{2}$ (HOMO-4 $\rightarrow$ LUMO+1, $441 \mathrm{~nm}$ ) (Fig. 3b). The low energy band consists of one electronic transition $\mathrm{S}_{1}(\mathrm{HOMO}-2 \rightarrow \mathrm{LUMO}+1,558 \mathrm{~nm})$. Compared with TpBpy, the low energy electronic transition in Re-TpBpy involves the excitation of the electron from the orbital in both Tp and Bpy moieties. The detailed calculated orbitals for both samples have been summarized in the supporting information.

\section{Time-resolved Photoluminescence}

In the next step, we studied the photoluminescence (PL) dynamics of the samples. The steady-state $\mathrm{PL}$ spectra of TpBpy and Re-TpBpy are identical in terms of emission energy and spectral shapes (Fig. 4a). However, the relative PL quantum yield (extracted from absorption calibrated PL intensities) of Re-TpBpy is much lower. This should be attributed to the PL quenching by the integration of the Re-complex. The shorter PL lifetime of Re-TpBpy measured from time-correlated single-photon counting (TCSPC) verify the additional non-radiative process (Fig.4b). The exponential fitting can resolve two components with lifetimes of $1.1 \mathrm{~ns}(91 \%), 19 \mathrm{~ns}$ (9\%) for TpBpy and 716ps (92\%), 40ns (8\%) for Re-TpBpy. However, the lifetime of the fast components (i.e. $1.1 \mathrm{~ns}$ for TpBpy and $716 \mathrm{ps}$ for Re-TpBpy) are limited by the response function in the TCSPC measurement. Therefore streak camera technique was employed to explore the ultra-fast process. A similar faster PL decay of Re-TpBpy than TpBpy can be observed in Fig.4c. The PL decays can be fitted by tri-exponential functions. The two fast components can then be fitted as 106 ps (74\%), 481 ps (24\%) for TpBpy and 98 ps (66\%), 340 ps (31\%) for Re-TpBpy.

\section{Femtosecond transient visible absorption spectroscopy.}


The excited-state dynamics of the samples were explored by transient absorption (TA) spectroscopy. We first excited the samples close to their band edge at $530 \mathrm{~nm}$. In this case, all the excited species should populate the lowest excited states instantly. The TA spectra of TpBpy exhibit one broad negative band (B1) from 450 to $595 \mathrm{~nm}$ attributed to the band-edge ground state bleach (GSB) together with two positive excited state absorption bands (ESA, A1 and A2) from $600 \mathrm{~nm}$ to $700 \mathrm{~nm}$ (Fig. 5a). According to the above DFT calculation in Fig. 3a, $530 \mathrm{~nm}$ excitation will only trigger the transition to the lowest excited state (i.e. HOMO to LUMO) in TpBpy. Hence, A1 and A2 here should not be attributed to different levels of the excited state. One possible explanation is the excited state transform from a normal exciton state (A2) to a polaron state (A1) where the excitons are self-trapped within the local structure of the $\mathrm{COF}^{29}$. Such polaron formation also complies with the significant stokes shift from the PL spectra, as shown in Fig. 2a.

For Re-TpBpy, only one B1 can be observed with the absence of long-lived ESA (Fig. 5b). This already indicates the charge transfer from the excited states. A more quantitative analysis was then implemented using singular value decomposition (SVD) fitting (Fig. 5a\&b, lower panel). The TA dynamics of TpBpy can be decomposed into four decay-associated components $\left(t_{1}-t_{4}\right)$. The first three components $\left(t_{1}-t_{3}\right)$ shared the same negative GSB signal with the identical position (B1), denoting the population of the lowest excited state. The difference between the first component $\left(t_{1}=2 \mathrm{ps}\right)$ with the second and third components ( $t_{2}=70 \mathrm{ps}$ and $t_{3}=4 \mathrm{~ns}$ ) appears as the blue-shifted ESA band from $A 2$ to $A 1$ by about 50 nm. TA kinetics at the A1 (Fig. 5d, blue curve) and A2 (Fig. 5e, blue curve) reveal the concurrent rising of $A 1$ and decay of $A 2$. This indicates the transformation of the lowest excited state (e.g. polaron formation) within 2 ps corresponding to the transition of ESA from A2 to A1 in TA spectra. Components 2 and 3 exhibits the same spectral feature corresponding to the depopulation dynamics of the same lowest excited state. The slowest component 4 featured as broad negative band with a lifetime exceeding the TA time window. This can also be visualized in the TA kinetics in Fig. 5c-e. After functionalization by the Re-complex, the TA dynamics of Re-TpBpy can also be decomposed into four components $\left(t_{1}-t_{4}\right)$ with lifetime of $t_{1}=990 \mathrm{fs}, t_{2}=13 \mathrm{ps}, \mathrm{t}_{3}=262 \mathrm{ps}$ accompany with one ultra-long component ((Fig. $\left.5 \mathrm{~b}\right)$. Component $t_{1}-t_{3}$ of Re-TpBpy resembles the GSB feature as TpBpy but with shorter lifetimes as evidenced by the comparison of B1 kinetics in Fig. 5c. Most importantly, ESA bands are completely absent in components $t_{2}$ and $t_{3}$. This suggests the ultrafast charge transfer from the lowest excited state. In addition, the longest component $t_{4}$ also exhibits narrower GSB at the band edge position compared with GSB in component $t_{4}$ of TpBpy.

In order to monitor the dynamics of hot carriers, high energy excitation has also been employed in both samples. Compared with TA spectra excited at $530 \mathrm{~nm}$, here the TA spectra of both TpBpy and Re-TpBpy (Fig.6a\&b) exhibit one additional negative band (B2) around $450 \mathrm{~nm}$ (Fig. 6) with the slight red-shifted B1 to $515 \mathrm{~nm}$. Since B2 appears in both TpBpy and Re-TpBpy, the additional bleach band should be attributed to the population of high energy/hot levels in the COFs unit. SVD fitting indicates that the dynamics of TpBpy can be described by four main components. The fastest component $t_{1}(2 \mathrm{ps})$ consists of $B 1, B 2$, and $A 1$. Component $t_{2}$ (34 ps) features the same $B 1$ and $B 2$ bands but the ESA is blue-shifted 
to $A 2$. The component $t_{3}(480 \mathrm{ps})$ lifetime shares almost the same spectral features of component 2 except for the absence of B2. A similar lifetime (481 ps) can also be extracted in the PL decay of TpBpy (Fig. 4c), manifesting the radiative recombination of the band-edge charge carriers. The component $t_{4}$ only contains B1 but the contribution is negligible. The above SVD analysis indicates the long-lived B1 versus short-lived B2 as also evidenced by the extracted TA kinetics in Fig. $6 \mathrm{c}$ and d (blue curve).

On the other hand, both A1 and A2 appear instantaneously which is different from the ones observed for $530 \mathrm{~nm}$ excitation. TA spectra of Re-TpBpy can also be fitted with four main components $\left(t_{1}=2 \mathrm{ps}, \mathrm{t}_{2}=\right.$ $24 \mathrm{ps}, \mathrm{t}_{3}=340 \mathrm{ps}$ and one ultra-long component). The features of component $\mathrm{t}_{1}$ resemble those of TpBpy with a similar lifetime of $2 \mathrm{ps}$. Compared with $530 \mathrm{~nm}$ excitation, B2 with $400 \mathrm{~nm}$ excitation are long-lived in both $t_{1}-t_{3}$ up to 340 ps. The prolonged B2 band in Re-TpBpy suggests that the long-lived high energy excited state population in contrast to TpBpy as further illustrated by the TA kinetics in Fig. $6 \mathrm{~d}$. Furthermore, $A 1$ disappears in component $t_{2}$ and reoccurs in $t_{3}$. This is also reflected by the different $A 1$ kinetics (Fig. 6e, red curve) compared with the B1 and B2 kinetics (Fig. 6c\&d, red curve) especially at the timescale between 5 to $20 \mathrm{ps}$. The absence of $A 1$ in component $t_{2}$ can be induced by two possible scenarios: 1) there exist two pools of Re-TpBpy where electron transfer from TpBpy to Re' occurs in one pool and absent in the other. 2) the charge transfer of hot electrons from the COFs to Rel centers is followed by the back-transfer to the LUMO level. In the following, we will demonstrate the latter is more likely evidenced by time-resolved IR spectroscopy results, which probes the transient population of electrons at $\mathrm{Re}^{\mathrm{l}}$ centers. The lifetime $t_{3}(340 \mathrm{ps})$ can be obtained from the TRPL decay in Fig. 4c, manifesting radiative recombination with hot carriers, which accounts for the high energy emission band in the steady-state PL spectrum (Fig. 2a). Identical to TpBpy, component 4 of Re-TpBpy comprises only B1 with negligible amplitude. In short, the additional B2 band and the wider ESA band when excited at $400 \mathrm{~nm}$ in components $\mathrm{t}_{1}, \mathrm{t}_{3}$ reflect the long-live hot excited level population. On the other hand, the absence of $A 1$ in component $t_{2}$ confirms the charge transfer of hot electrons to Rel centers within $2 \mathrm{ps}$.

\section{Femtosecond transient infrared absorption spectroscopy}

In order to further characterize the excited state dynamics at the two excitation wavelengths, we measured the time-resolved IR (TRIR) spectra of the samples. TRIR can probe photo-induced electronic transitions at low energy such as molecular vibrations or intraband free carriers ${ }^{47-51}$. No TRIR signal can be observed in TpBpy and Re-Bpy when excited at $530 \mathrm{~nm}$ (Fig.7a and Fig. 7b). However, the TRIR spectrum of Re-TpBpy exhibits pronounced differential dips at $1850 \mathrm{~cm}^{-1}$ and $2040 \mathrm{~cm}^{-1}$ (Fig. 7c), resembling the spectral feature of pure Re-Bpy excited at $400 \mathrm{~nm}$ (Fig.7f). Such differential dips are the fingerprint features of excited $\left[\operatorname{Re}^{\prime}(b p y)(C O) 3\right]^{*}$ as the $\mathrm{C}-0$ stretching vibration is perturbed due to the formation of Re radical species ${ }^{52}$. The TRIR kinetics in Fig. $7 d$ suggests that the Re' radical is formed within $0.6 \mathrm{ps}$ (rising time of the kinetics) together with 2 decay lifetimes (15 ps and $2.3 \mathrm{~ns}$ ). Such formation time of the Rel radical is consistent with $t_{1}$ in TA components ( 0.99 ps) (Fig. 5b), confirming the sub-picosecond electron transfer from TpBpy to the Rel center after excitation. The 15 ps decay lifetime is 
identical to the component $\mathrm{t}_{3}$ in TA (Fig. $5 \mathrm{~b}$ ). When excited at $400 \mathrm{~nm}$, the TRIR spectra of both TpBpy and Re-TpBpy are dominated by the featureless positive absorption (Fig. 7 e\&g), which is widely accepted as the sign of free carrier generation in semiconductor materials ${ }^{47,53,54}$. This means that the hot excited states reflected by the $\mathrm{B} 2$ and the broad $\mathrm{A} 1$ band in TA should all be populated by free carriers when excited at $400 \mathrm{~nm}$. Moreover, the TRIR spectrum in Re-TpBpy features additional differential dips of the $\mathrm{Re}^{\mathrm{l}}$ radical, indicating the COFs-Re electron transfer occurs. We can decompose the dynamics of $\mathrm{Re}^{\mathrm{l}}$ radical (orange curve, Fig. $7 \mathrm{~h}$ ) by subtracting the TA kinetics at such mixed region $\left(2040 \mathrm{~cm}^{-1}\right.$, red curve, Fig. $7 \mathrm{~h}$ ) by the kinetics at the region only showing positive absorption $\left(1850 \mathrm{~cm}^{-1}\right.$, blue curve, Fig. $\left.7 \mathrm{~h}\right)$. Here the intensity of TA kinetics at $1850 \mathrm{~cm}^{-1}$ is scaled up by the amplitude ratio between $1850 \mathrm{~cm}^{-1}$ and $2040 \mathrm{~cm}^{-1}$ as extracted in Fig. $7 \mathrm{~g}\left(\mathrm{~A}_{2040 \mathrm{~cm}-1} / \mathrm{A}_{1850 \mathrm{~cm}-1}=1.9\right)$ with only free carrier contribution in COFs. The deferential kinetics (i.e. the orange curve in Fig. $7 \mathrm{H}$ ) shows a 0.8 ps building up time followed by a 26 ps decay, which is consistent with the above argumentation that the hot electrons are injected to $\mathrm{Re}^{\mathrm{l}}$ center within the picosecond and rebounce to the $S_{1}$ level of TpBpy in $26 \mathrm{ps}$. We also notice that such kinetics is different from the depopulation of photo-excited pure $\left[\operatorname{Re}^{\prime}(b p y)(C O) 3\right]^{\star}$ (green curve, Fig. 7h). This means the back transfer or geminate recombination of injected electrons in $\mathrm{Re}^{\mathrm{l}}$ center is faster than the electron-hole recombination in the Re'(bpy) moiety.

\section{Discussion}

\section{Cold Electron Injection}

The TD-DFT calculations indicate the $530 \mathrm{~nm}$ excitation can only generate an electronic transition from HOMO to LUMO $\left(\mathrm{S}_{1}\right)$ in TpBpy, which merely locates at Bpy moiety (Fig. 3a). The TRIR results further suggest that the dominant excited species are excitons. Such bounded exciton formation is reasonable as the photo-generated electrons and holes are close in space in Bpy. The ESA transition from A2 to A1 in TA spectra in Fig. 5a should represent the formation of exciton polarons from initially generated excitons. The following up excited state depopulation at hundreds of ps can be observed in both the TA and TRPL results. We confirmed that the lifetime of such a process is highly dependent upon the excitation intensity (for details, see S8b). This indicates that the high order recombination of excited singlet excitons, which is often observed in conjugated polymers and semiconductor nanostructrues ${ }^{55-58}$. Previous research reports such ultrafast singlet-singlet exciton annihilation in COFs materials followed by the formation of ultra-long-lived specially separated charges ${ }^{30}$. However, the absence of free carriers over the whole timewindow suggests that the residual excited state species are still excitons instead of free carriers in our COF. However, the lifetime of such excitons varies according to the relative spatial location. The short lifetime (4 ns) should refer to the excitonic recombination within the same COFs sheet, while the ultralong lived exciton may contain the electrons and holes at a different sheet of $\mathrm{COFs}^{30,59}$. Fig. 8a summarizes the observed excited-state dynamics. 
In Re-TpBpy, the electronic transition from the HOMO-2 to the LUMO (Fig. 3b) corresponds to a partial charge transfer state as the HOMO-2 locates at the orbitals over both Tp and Bpy moiety according to the calculation. The sub-picosecond depopulation of excited states in the TA spectra combined with rising of transient Re* radical formation spectra in TRIR can be unambiguously assigned to the electron transfer from the LUMO located at Bpy to the Rel center within 0.8-0.9 ps as shown in Fig. 8b. However, as holes can reside both at $\mathrm{Tp}$ and Bpy, we can expect different depopulation pathways of those holes after electron injection corresponding to two-lifetime components in TA ( $t_{2}$ and $t_{3}$ ) (Fig. 5b). Holes at Bpy should undergo fast germinate recombination with electrons at Rel center due to the short distance between electrons and holes (13 ps), which is consistent with the same decay component (15 ps) in TRIR (Fig. 7d) demonstrating the concurrent depopulation of electrons. On the other hand, those holes at Tp should be implausible to recombine with remote electrons at Rel directly,instead, they will cool down to the HOMO level with relatively long time (262 ps) which is still strongly localized in Tp (for detailed electronic structure see Table S3). Such slow cooling time may be due to the necessary inter-unit (e.g. Tp to Bpy, or Bpy to $\mathrm{Tp}$ ) charge transfer in the cooling pathway. Therefore recombination between those holes and electrons in Re center will be inefficient, corresponding to the long component in both TA (Fig. 5b) and TRIR (Fig. 7d). The above analysis suggests the low energy excitation in Re-TpBpy is very close to the Re-COF interface, thus facilitating the electron injection from the COFs to the Re' center. However, geminate recombination is also efficient due to the close spacing between the injected electron and residual holes in COF moiety, as summarized in Fig. 8b.

\section{Hot Electron Injection}

TD-DFT calculation demonstrates at high-energy photon excitation condition, TpBpy will have a transition from $\mathrm{HOMO} \rightarrow \mathrm{LUMO}+2$ and $\mathrm{HOMO}-3 \rightarrow$ LUMO+1 (Fig. 3a), while Re-TpBpy will exhibit an electronic transition from $\mathrm{HOMO} \rightarrow \mathrm{LUMO}+2$ and $\mathrm{HOMO}-4 \rightarrow \mathrm{LUMO}+1$ (Fig. 3b). Compared with low energy excitation, the excited states are distributed more evenly through the Tp and Bpy. In addition, the TRIR results suggest that the initially excited species include free carriers resided at those high energy levels. The initial fastest component $\mathrm{t}_{1}$ in TA measurement (Fig. 6a) should be related to the partial polaron formation where some of the generated species also remain as free carriers as evidenced by the remaining A2 after 2 ps and the free carries absorption in the TRIR spectra. The dual emission band in steady state PL of Fig. 2a supports that the excited state depopulation should involve two parallel processes with radiative recombination from the higher level and lowest excited state. In TpBpy, the population of hot electrons at the LUMO+2 and the LUMO+1 as well as hot holes at the HOMO-3 contributed to the B2 in Fig. 6a while hole population at HOMO leads to the B1 consistent with the band edge GSB at $530 \mathrm{~nm}$ excitation. $\mathrm{t}_{2}$ component in TA (Fig. 6a) features concurrent B1 and B2 should then be attributed to the radiative recombination between the electron at LUMO+1 and hole at HOMO-3 leading to the high energy PL emission. $T_{3}$ component, on the other hand, should be attributed to the recombiniation bewteen electrons and holes relaxed to the HOMO and LUMO level. Fig. 8c summarized the state dynamics. 
As summarized in Fig. 8d, at Re-TpBpy, the excited electron at LUMO+2 and LUMO+1 would be injected to $\mathrm{Re}^{\mathrm{l}}$ center within $2 \mathrm{ps}$ and then recombines to HOMO at Bpy within $24 \mathrm{ps}$ resolved by the complementary dynamics observed in the TA and TRIR measurements, as shown in Fig. 8d. This can be due to either the hot electron injection through the higher excited states in Rel center or to the formation of a chargetransfer state (CTS) due to the Coloumbic attraction between injection electron in $\mathrm{Re}^{\mathrm{l}}$ and residual hole in COF followed by the dissociation of such CTS. Both pathways are well-observed in other molecular or semiconductor systems ${ }^{60,61}$. Such a 24 ps lifetime for such intermediate excited state can be a merit for hot-carrier harvesting. After hot carrier cooling, the depopulation of the excited states also depends on the spatial location of the charge carriers. If the hole locates at Bpy orbitals ( $\mathrm{HOMO}-4$ level at $\mathrm{S}_{2}$ in Fig. $3 \mathrm{~b}$ ), the hot carrier emission will occur similarly to the case of TpBpy with lifetime of 340 ps corresponding to the $t_{3}$ component observed in TA (Fig. 6b), since the hot hole cooling to the HOMO in Re-TpBpy is hindered by the additional Bpy-to-Tp charge transfer. If hole locates at Tp orbitals in $\mathrm{HOMO}\left(\mathrm{S}_{3}\right.$ in Fig. $\left.3 \mathrm{~b}\right)$, it will recombine with the relaxed electron at LUMO both radiatively and nonradiatively but with a longer lifetime as observed in both the TA and TRPL spectras.

\section{Influence on the photocatalytic reaction:}

The above analysis on the excited state dynamics of TpBpy and Re-TpBpy suggest that anchoring Recomplex into COFs structure does facilitate the charge separation for the photocatalytic reduction process. However, when high excitation photon energy is used, efficient hot electron injection would occur from TpBpy to higher energy orbital of Re-complex with the electron lifetime at Re' center almost doubled compared to the lifetime with $530 \mathrm{~nm}$ excitation. In contrast to the conventional semiconductor where the photo-generated hot electrons will quickly dissipate energy and relax to the lowest excited state, the unique electronic structure of the Re-TpBpy catalyst is expected to boost the photocatalytic performance from hot carriers. The following $\mathrm{CO}_{2}$ photocatalytic reduction experiment confirmed our assumption. The evolution of $\mathrm{CO}$ by our Re-COFs catalyst exhibit a much higher yield when $440 \mathrm{~nm}$ excitation is used compared with band-edge excitation at $520 \mathrm{~nm}$ (Fig. 9a), which should be attributed to 1) injected electrons are located at high energy levels in Rel centers with longer lifetime which favorable for the electron transfer process for the $\mathrm{CO}_{2}$ reduction, and 2) when excited with high energy besides the injected electrons to $\mathrm{Re}^{\mathrm{l}}$ center, there still remain long-lived electrons in COF moiety which is transferred back from Rel. This makes the two-electron reaction of $\mathrm{CO}_{2}$ conversion to $\mathrm{CO}$ to work as illustrated in Fig. $9 b$.

\section{Conclusion}

In this paper, we investigated the excited state dynamics with focus on the ultrafast charge transfer in $\operatorname{Re}^{\prime}(\mathrm{bpy})(\mathrm{CO})_{3} / \mathrm{TpBpy}$ hybrid photocatalyst by complementary time-resolved laser spectroscopies and numerical methods. We first determine the electronic transition of the hybrid structure using timedependent DFT calculations to model the optical absorption. We found that the absorption spectrum of Re-TpBpy mainly consists of two bands with the low energy bands contributed by the transition from 
ground state to excited state barely in Bpy moiety and the high-energy bands features the unoccupied orbital is contributed evenly by the whole COFs moiety. Combining the observations of the excited dynamics resolved both in TA and TRIR, entirely different inter-unit charge transfer pathways in Re-TpBpy can be identified. Under band-edge excitation, the electrons excited at the HOMO level would quickly injected into $\operatorname{Re}^{\prime}(\mathrm{bpy})(\mathrm{CO})_{3}$ within ps timescale and recombine within ns with the holes residing in Bpy close to Re center at about 13 ps. Under excitation with high energy photon, the photo-generated hotelectron is first injected into the highly excited level of $\operatorname{Re}^{\prime}(\mathrm{bpy})(\mathrm{CO})_{3}$ within 1-2 ps and recombine to the $\mathrm{HOMO}$ in COF within 24 ps. In addition, there remains long-lived free carriers in the COF moiety. This can rationalize the good photocatalytic $\mathrm{CO}_{2}$ reduction performance of the obtained catalysts.

\section{References}

1. Song, C. Global challenges and strategies for control, conversion and utilization of $\mathrm{CO} 2$ for sustainable development involving energy, catalysis, adsorption and chemical processing. Catal. Today 115, 2-32 (2006).

2. Omae, I. Aspects of carbon dioxide utilization. Catal. Today 115, 33-52 (2006).

3. Wu, J., Huang, Y., Ye, W. \& Li, Y. CO2 Reduction: From the Electrochemical to Photochemical Approach. Adv. Sci. 4, 1700194 (2017).

4. Tu, W., Zhou, Y. \& Zou, Z. Photocatalytic Conversion of $\mathrm{CO} 2$ into Renewable Hydrocarbon Fuels: State-of-the-Art Accomplishment, Challenges, and Prospects. Adv. Mater. 26, 4607-4626 (2014).

5. INOUE, T., FUJISHIMA, A., KONISHI, S. \& HONDA, K. Photoelectrocatalytic reduction of carbon dioxide in aqueous suspensions of semiconductor powders. Nature 277, 637-638 (1979).

6. Wang, C., Sun, Z., Zheng, Y. \& Hu, Y. H. Recent progress in visible light photocatalytic conversion of carbon dioxide. J. Mater. Chem. A 7, 865-887 (2019).

7. Roy, S. C., Varghese, O. K., Paulose, M. \& Grimes, C. A. Toward Solar Fuels: Photocatalytic Conversion of Carbon Dioxide to Hydrocarbons. ACS Nano 4, 1259-1278 (2010).

8. Takeda, H. \& Ishitani, O. Development of efficient photocatalytic systems for $\mathrm{CO} 2$ reduction using mononuclear and multinuclear metal complexes based on mechanistic studies. Coord. Chem. Rev. 254, 346-354 (2010).

9. Schneider, T. W., Ertem, M. Z., Muckerman, J. T. \& Angeles-Boza, A. M. Mechanism of Photocatalytic Reduction of $\mathrm{CO} 2$ by $\operatorname{Re}(\mathrm{bpy})(\mathrm{CO}) 3 \mathrm{Cl}$ from Differences in Carbon Isotope Discrimination. ACS Catal. 6, 5473-5481 (2016).

10. Yamazaki, Y., Takeda, H. \& Ishitani, O. Photocatalytic reduction of $\mathrm{CO} 2$ using metal complexes. J. Photochem. Photobiol. C Photochem. Rev. 25, 106-137 (2015).

11. Hori, H., Johnson, F. P. A., Koike, K., Ishitani, O. \& Ibusuki, T. Efficient photocatalytic CO2 reduction using $[\operatorname{Re}(\mathrm{bpy})(\mathrm{CO}) 3\{\mathrm{P}(\mathrm{OEt}) 3\}]+$. J. Photochem. Photobiol. A Chem. 96, 171-174 (1996).

12. Hawecker, J., Lehn, J.-M. \& Ziessel, R. Efficient photochemical reduction of $\mathrm{CO} 2$ to $\mathrm{CO}$ by visible light irradiation of systems containing $\operatorname{Re}($ bipy)(CO) $3 \mathrm{X}$ or $\mathrm{Ru}(\mathrm{bipy}) 32+-\mathrm{Co} 2+$ combinations as 
homogeneous catalysts. J. Chem. Soc. Chem. Commun. 536-538 (1983) doi:10.1039/C39830000536.

13. Takeda, H., Koike, K., Morimoto, T., Inumaru, H. \& Ishitani, O. Photochemistry and photocatalysis of rhenium(I) diimine complexes. in Inorganic Photochemistry (eds. Eldik, R. van \& Stochel, G. B. T.-A. in I. C.) vol. 63 137-186 (Academic Press, 2011).

14. Koike, K. et al. Key Process of the Photocatalytic Reduction of $\mathrm{CO} 2$ Using $\left[\operatorname{Re}\left(4,4^{\prime}-\mathrm{X} 2\right.\right.$-bipyridine) (CO)3PR3]+ (X = CH3, H, CF3; PR3 = Phosphorus Ligands): Dark Reaction of the One-ElectronReduced Complexes with CO2. Organometallics 16, 5724-5729 (1997).

15. Yamazaki, Y., Takeda, H. \& Ishitani, O. Photocatalytic reduction of $\mathrm{CO} 2$ using metal complexes. Journal of Photochemistry and Photobiology C: Photochemistry Reviews vol. 25 106-137 (2015).

16. Vlček, A. Ultrafast Excited-State Processes in Re(I) Carbonyl-Diimine Complexes: From Excitation to Photochemistry BT - Photophysics of Organometallics. in (ed. Lees, A. J.) 115-158 (Springer Berlin Heidelberg, 2010). doi:10.1007/3418_2009_4.

17. Qiao, X. et al. Well-Defined Nanographene-Rhenium Complex as an Efficient Electrocatalyst and Photocatalyst for Selective CO2 Reduction. J. Am. Chem. Soc. 139, 3934-3937 (2017).

18. Huang, R., Peng, Y., Wang, C., Shi, Z. \& Lin, W. A Rhenium-Functionalized Metal-Organic Framework as a Single-Site Catalyst for Photochemical Reduction of Carbon Dioxide. Eur. J. Inorg. Chem. 2016, 4358-4362 (2016).

19. Faustino, L. A. et al. Photocatalytic $\mathrm{CO} 2$ Reduction by $\operatorname{Re}(\mathrm{I})$ Polypyridyl Complexes Immobilized on Niobates Nanoscrolls. ACS Sustain. Chem. Eng. 6, 6073-6083 (2018).

20. Windle, C. D. et al. Improving the Photocatalytic Reduction of $\mathrm{CO} 2$ to $\mathrm{CO}$ through Immobilisation of a Molecular Re Catalyst on TiO2. Chem. - A Eur. J. 21, 3746-3754 (2015).

21. Xu, R. et al. Rhenium-modified porous covalent triazine framework for highly efficient photocatalytic carbon dioxide reduction in a solid-gas system. Catal. Sci. Technol. 8, 2224-2230 (2018).

22. Yang, S. et al. 2D Covalent Organic Frameworks as Intrinsic Photocatalysts for Visible Light-Driven C02 Reduction. J. Am. Chem. Soc. 140, 14614-14618 (2018).

23. Fu, Z. et al. A stable covalent organic framework for photocatalytic carbon dioxide reduction. Chem. Sci. 11, 543-550 (2020).

24. Li, S.-Y. et al. Rhenium-functionalized covalent organic framework photocatalyst for efficient $\mathrm{CO} 2$ reduction under visible light. Microporous Mesoporous Mater. 285, 195-201 (2019).

25. Côté, A. P. et al. Porous, Crystalline, Covalent Organic Frameworks. Science (80-. ). 310, 1166 LP 1170 (2005).

26. Huang, N., Wang, P. \& Jiang, D. Covalent organic frameworks: a materials platform for structural and functional designs. Nat. Rev. Mater. 1, 16068 (2016).

27. Hu, X.-L., Li, H.-G. \& Tan, B.-E. COFs-based Porous Materials for Photocatalytic Applications. Chinese J. Polym. Sci. 38, 673-684 (2020). 
28. Yang, Q., Luo, M., Liu, K., Cao, H. \& Yan, H. Covalent organic frameworks for photocatalytic applications. Appl. Catal. B Environ. 276, 119174 (2020).

29. Kim, T. W. et al. Ultrafast charge transfer coupled with lattice phonons in two-dimensional covalent organic frameworks. Nat. Commun. 10, 1873 (2019).

30. Jakowetz, A. C. et al. Excited-State Dynamics in Fully Conjugated 2D Covalent Organic Frameworks. J. Am. Chem. Soc. 141, 11565-11571 (2019).

31. Nozik, A. J. Utilizing hot electrons. Nat. Energy 3, 170-171 (2018).

32. Sun, Y. \& Tang, Z. Photocatalytic hot-carrier chemistry. MRS Bull. 45, 20-25 (2020).

33. Nozik, A. J. Quantum dot solar cells. Phys. E Low-dimensional Syst. Nanostructures 14, 115-120 (2002).

34. Zhang, C. et al. Hot-carrier transfer at photocatalytic silicon/platinum interfaces. J. Chem. Phys. 152, 144705 (2020).

35. Harutyunyan, H., Suchanek, F., Lemasters, R. \& Foley, J. J. Hot-carrier dynamics in catalysis. MRS Bull. 45, 32-36 (2020).

36. Zhao, W., Shao, H., Yu, G., Hou, Y. \& Wang, S. The Coordination and Luminescence of the Eu(III) Complexes with the Polymers (PMMA, PVP). Polymers vol. 10 (2018).

37. Yang, L. et al. Theoretical Studies of Ground and Excited Electronic States in a Series of Halide Rhenium(I) Bipyridine Complexes. J. Phys. Chem. A 108, 6797-6808 (2004).

38. Aiyappa, H. B., Thote, J., Shinde, D. B., Banerjee, R. \& Kurungot, S. Cobalt-Modified Covalent Organic Framework as a Robust Water Oxidation Electrocatalyst. Chem. Mater. 28, 4375-4379 (2016).

39. Knopf, K. M. et al. In Vitro Anticancer Activity and in Vivo Biodistribution of Rhenium(I) Tricarbonyl Aqua Complexes. J. Am. Chem. Soc. 139, 14302-14314 (2017).

40. Haldar, S. et al. Anthracene-Resorcinol Derived Covalent Organic Framework as Flexible White Light Emitter. J. Am. Chem. Soc. 140, 13367-13374 (2018).

41. Agarwal, J., Fujita, E., Schaefer, H. F. \& Muckerman, J. T. Mechanisms for CO Production from CO2 Using Reduced Rhenium Tricarbonyl Catalysts. J. Am. Chem. Soc. 134, 5180-5186 (2012).

42. Tao, Y., Zou, W., Luo, G.-G. \& Kraka, E. Describing Polytopal Rearrangement Processes of Octacoordinate Structures. I. Renewed Insights into Fluxionality of the Rhenium Polyhydride Complex ReH5(PPh3)2(Pyridine). Inorg. Chem. 60, 2492-2502 (2021).

43. Zhao, Y. \& Truhlar, D. G. A new local density functional for main-group thermochemistry, transition metal bonding, thermochemical kinetics, and noncovalent interactions. J. Chem. Phys. 125, 194101 (2006).

44. Zhao, Y. \& Truhlar, D. G. The M06 suite of density functionals for main group thermochemistry, thermochemical kinetics, noncovalent interactions, excited states, and transition elements: two new functionals and systematic testing of four M06-class functionals and 12 other function. Theor. Chem. Acc. 120, 215-241 (2008). 
45. Weigend, F. \& Ahlrichs, R. Balanced basis sets of split valence, triple zeta valence and quadruple zeta valence quality for $\mathrm{H}$ to Rn: Design and assessment of accuracy. Phys. Chem. Chem. Phys. 7, 32973305 (2005).

46. Weigend, F. Accurate Coulomb-fitting basis sets for $\mathrm{H}$ to Rn. Phys. Chem. Chem. Phys. 8, 1057-1065 (2006).

47. Paz, Y. Transient IR spectroscopy as a tool for studying photocatalytic materials. J. Phys. Condens. Matter 31, 503004 (2019).

48. Furuhashi, K., Jia, Q., Kudo, A. \& Onishi, H. Time-Resolved Infrared Absorption Study of SrTiO3 Photocatalysts Codoped with Rhodium and Antimony. J. Phys. Chem. C 117, 19101-19106 (2013).

49. Munson, K. T., Kennehan, E. R. \& Asbury, J. B. Structural origins of the electronic properties of materials via time-resolved infrared spectroscopy. J. Mater. Chem. C 7, 5889-5909 (2019).

50. Takeshita, K. et al. Effect of Annealing Temperature on Back Electron Transfer and Distribution of Deep Trap Sites in Dye-Sensitized Ti02, Studied by Time-Resolved Infrared Spectroscopy. J. Phys. Chem. B 108, 2963-2969 (2004).

51. Heimer, T. A. \& Heilweil, E. J. Direct Time-Resolved Infrared Measurement of Electron Injection in DyeSensitized Titanium Dioxide Films. J. Phys. Chem. B 101, 10990-10993 (1997).

52. Abdellah, M. et al. Time-Resolved IR Spectroscopy Reveals a Mechanism with TiO2 as a Reversible Electron Acceptor in a TiO2-Re Catalyst System for CO2 Photoreduction. J. Am. Chem. Soc. 139, 1226-1232 (2017).

53. Gaubas, E., Simoen, E. \& Vanhellemont, J. Review-Carrier Lifetime Spectroscopy for Defect Characterization in Semiconductor Materials and Devices. ECS J. Solid State Sci. Technol. 5, P3108P3137 (2016).

54. Munson, K. T., Grieco, C., Kennehan, E. R., Stewart, R. J. \& Asbury, J. B. Time-Resolved Infrared Spectroscopy Directly Probes Free and Trapped Carriers in Organo-Halide Perovskites. ACS Energy Lett. 2, 651-658 (2017).

55. Sun, C., Figge, F., McGuire, J. A., Li, Q. \& Li, L. Biexciton Auger Recombination in Colloidal Graphene Quantum Dots. Phys. Rev. Lett. 113, 107401 (2014).

56. Makarov, N. S. et al. Spectral and Dynamical Properties of Single Excitons, Biexcitons, and Trions in Cesium-Lead-Halide Perovskite Quantum Dots. Nano Lett. 16, 2349-2362 (2016).

57. Žídek, K. et al. Ultrafast Dynamics of Multiple Exciton Harvesting in the CdSe-ZnO System: Electron Injection versus Auger Recombination. Nano Lett. 12, 6393-6399 (2012).

58. Brüggemann, B., Herek, J. L., Sundström, V., Pullerits, T. \& May, V. Microscopic Theory of Exciton Annihilation: Application to the LH2 Antenna System. J. Phys. Chem. B 105, 11391-11394 (2001).

59. Meng, Z., Stolz, R. M. \& Mirica, K. A. Two-Dimensional Chemiresistive Covalent Organic Framework with High Intrinsic Conductivity. J. Am. Chem. Soc. 141, 11929-11937 (2019).

60. Žídek, K., Abdellah, M., Zheng, K. \& Pullerits, T. Electron relaxation in the CdSe quantum dot - ZnO composite: prospects for photovoltaic applications. Sci. Rep. 4, 7244 (2014). 
61. Žídek, K. et al. Electron Transfer in Quantum-Dot-Sensitized ZnO Nanowires: Ultrafast Time-Resolved Absorption and Terahertz Study. J. Am. Chem. Soc. 134, 12110-12117 (2012).

\section{Declarations}

\section{Acknowledgements}

This work was supported by the Independent Research Fund Denmark-Sapere Aude starting grant (no. 7026-00037A), Swedish Research Council VR starting grant (no. 201705337), Crafood foundation (no.20200522), Swedish Energy Agency research grant, Research Fund for International Young Scientists from NSFC, China (no. 21950410515), and the Chinese Scholarship Council for the PhD scholarship to Q.Y., W.H., Q.Z and M. J.

M. A. H. acknowledges the financial support from the Swedish Research Council (VR) under project No. 2018-05393. M. A.-H. acknowledges support by the European Union via grant no. 824109 (European Microkelvin Platform) and by the Arab-German Young Academy of Sciences and Humanities (AGYA).

\section{Figures}

A

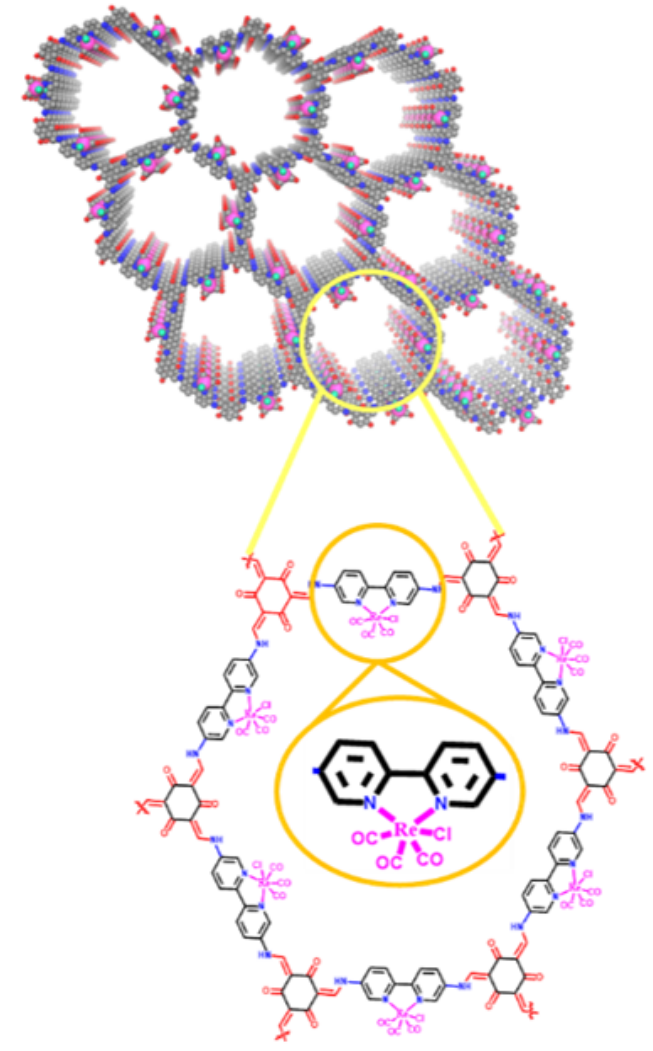

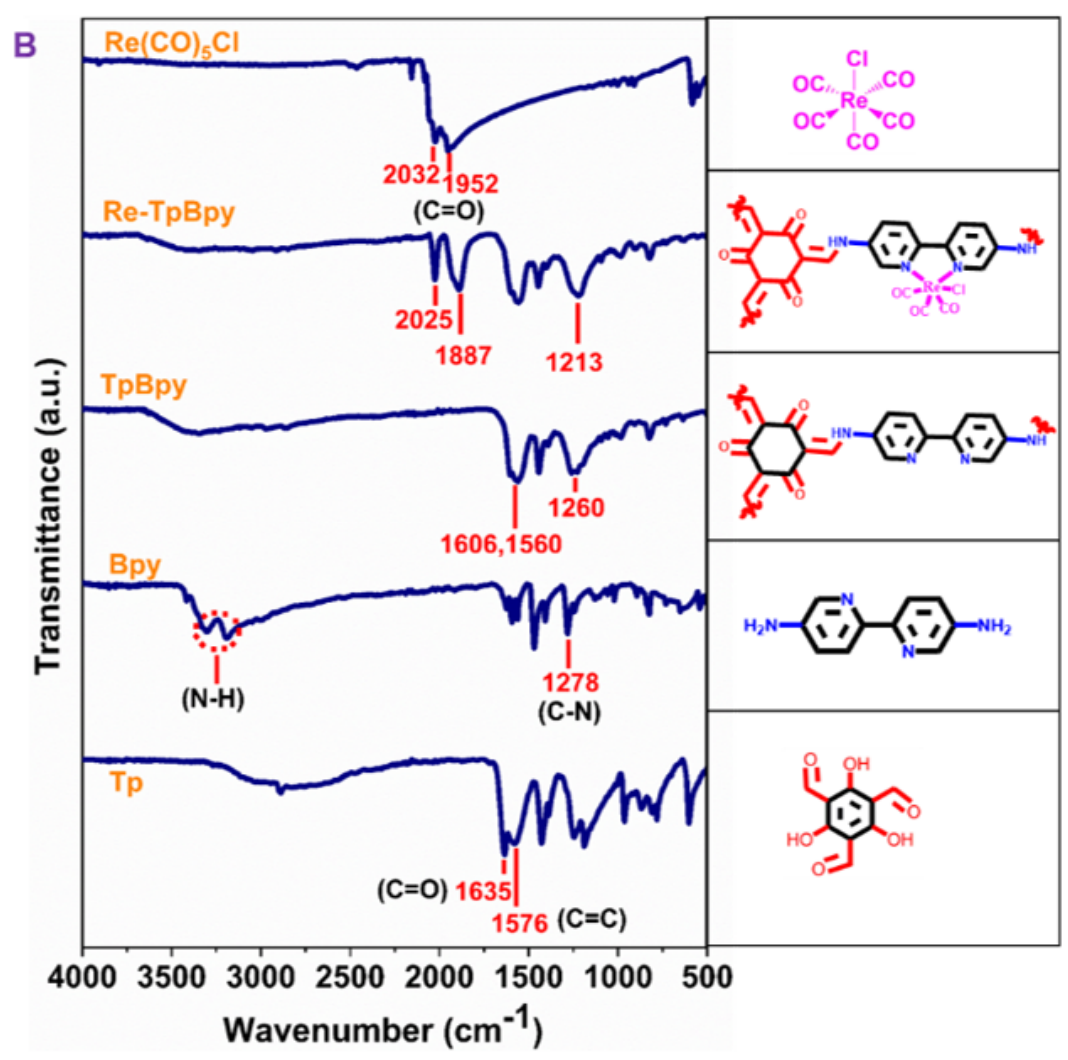

Figure 1 
(a) Schematic structure of the Re-TpBpy; (b) FT-IR spectra of TpBpy, Re-TpBpy and their starting materials with the right panel showing the molecular structure of the corresponding unit.
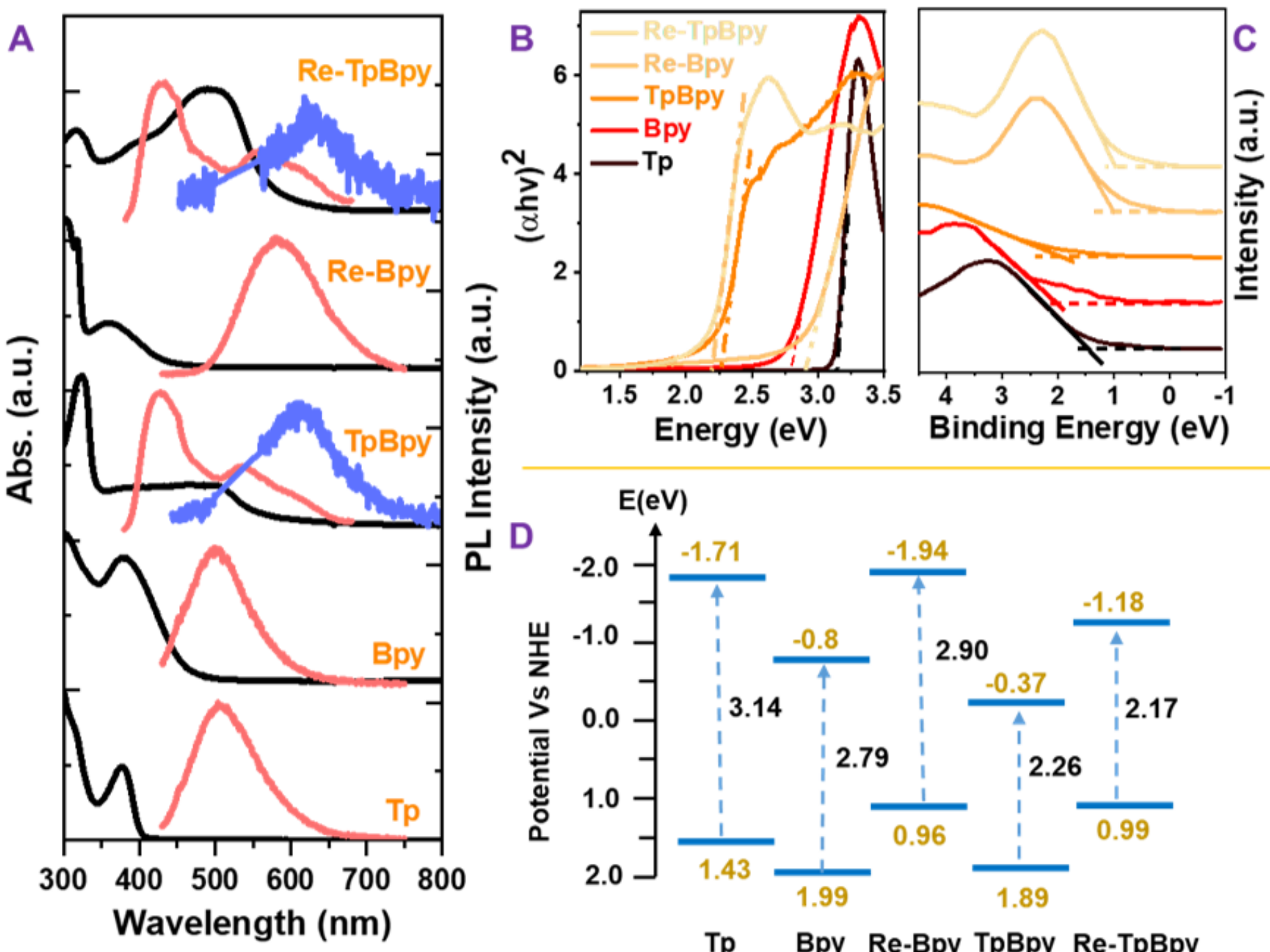

Tp Bpy Re-Bpy TpBpy Re-TpBpy

Figure 2

(a) Normalized UV-vis absorption (black) and steady-state photoluminescence spectra excited at $400 \mathrm{~nm}$ (red) and $530 \mathrm{~nm}$ (blue) of TpBpy, Re-TpBpy and their starting materials dispersed in Nafion (5\% w/w in water and 1-propanol); (b) Tauc plots of the absorption spectra determining the optical band gap of TpBpy, Re-TpBpy, and their starting materials; (c) XPS VB spectra, and (d) band alignment established from XPS and UV-vis measurement of TpBpy, Re-TpBpy, and their starting materials. 

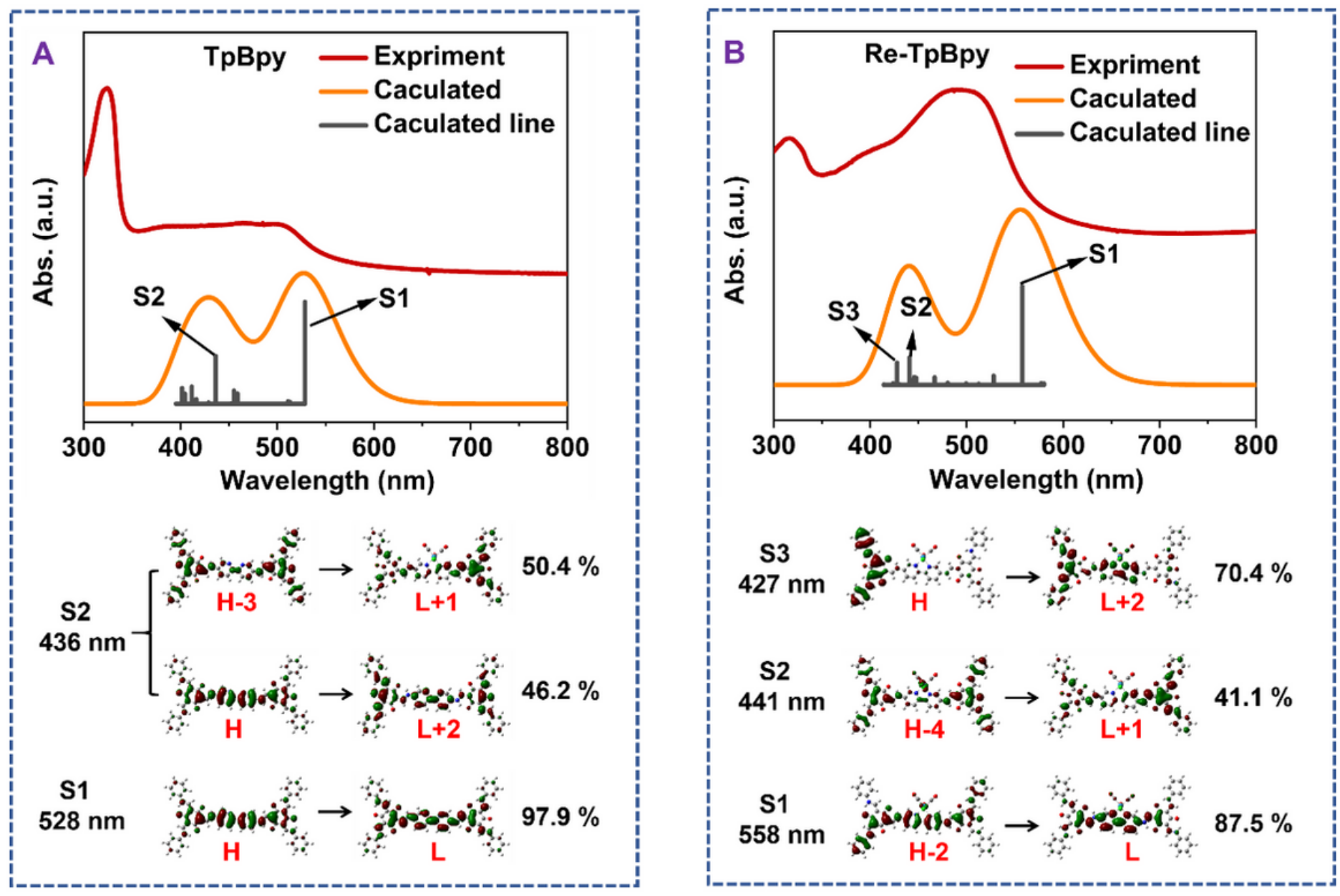

Figure 3

UV-vis absorption spectra of a) TpBpy and b) Re-TpBpy compared with TD-DFT calculated fragment.
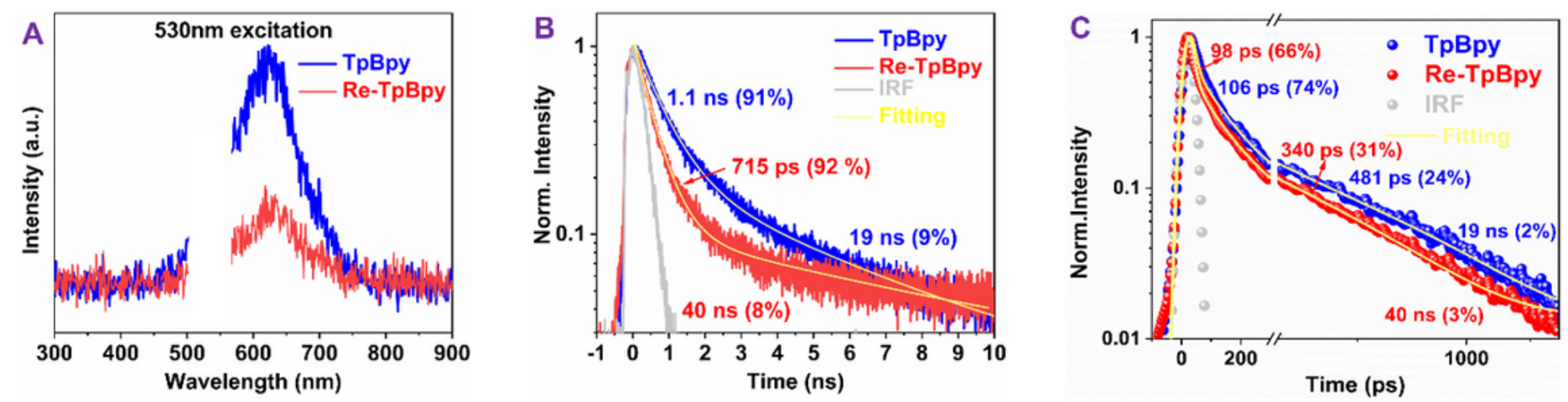

Figure 4

(a) Steady state PL emission spectra of TpBpy and Re-TpBpy normalized according to the absorbance at the excitation wavelength; (b) PL decays measured in TCSPC of the TpBpy and Re-TpBpy. Excitation wavelength $=438 \mathrm{~nm}$; (c) PL decays of the TpBpy and Re-TpBpy measured with streak camera excited at $400 \mathrm{~nm}$. 

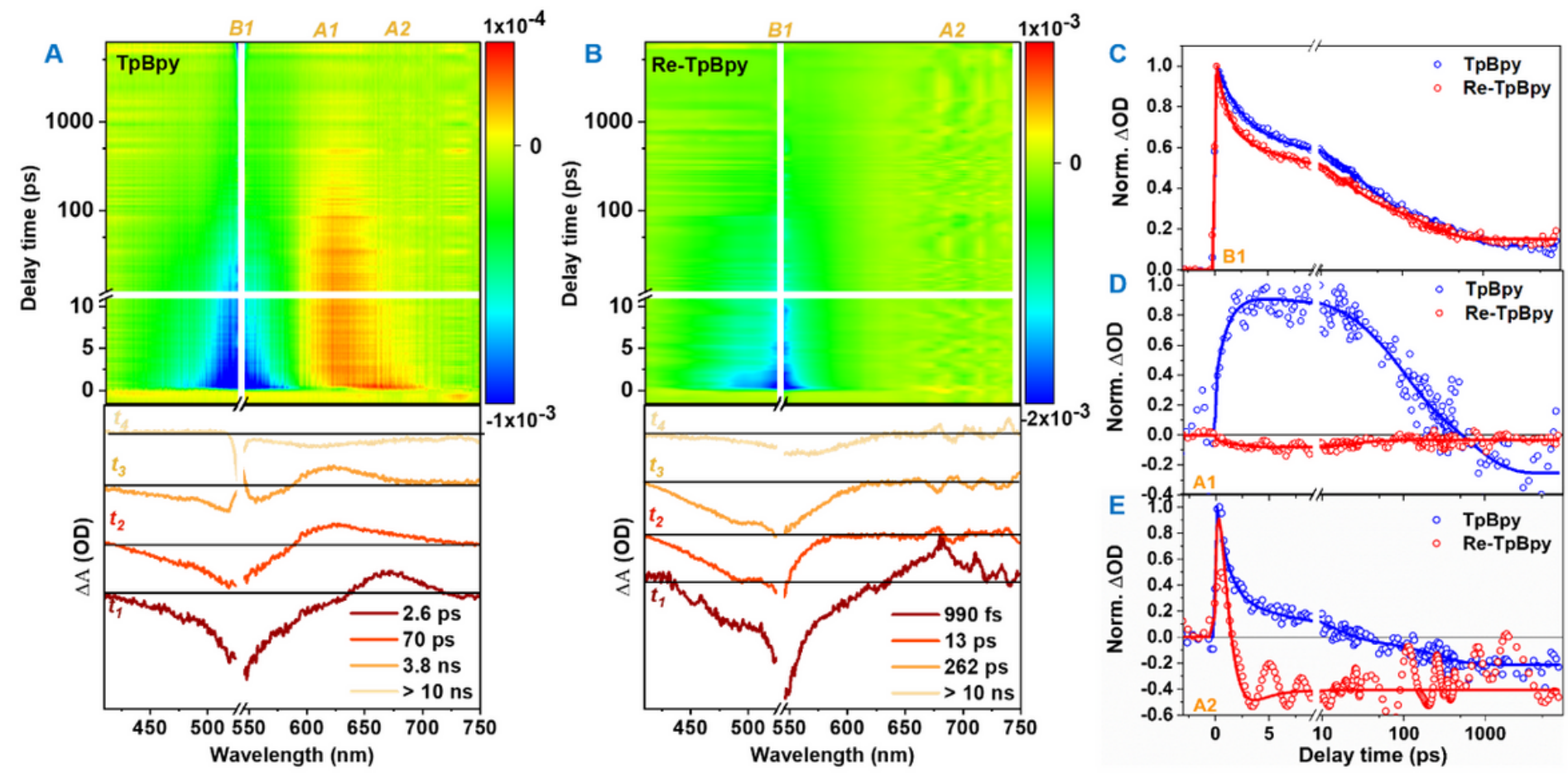

Figure 5

Transient absorption (TA) spectra under $530 \mathrm{~nm}$ excitation at the fluence of $2 \times 1013 \mathrm{ph} / \mathrm{cm} 2$ and the respective SVD fitting results of TpBpy (a), and Re-TpBpy (b). TA kinetics at some characteristic wavelength of B1 at $535 \mathrm{~nm}$ (c), A1 at $625 \mathrm{~nm}$ (d), and A2 at $675 \mathrm{~nm}(\mathrm{e})$. All the samples were measured in Nafion (5\% w/w in water and 1-propanol) solution.
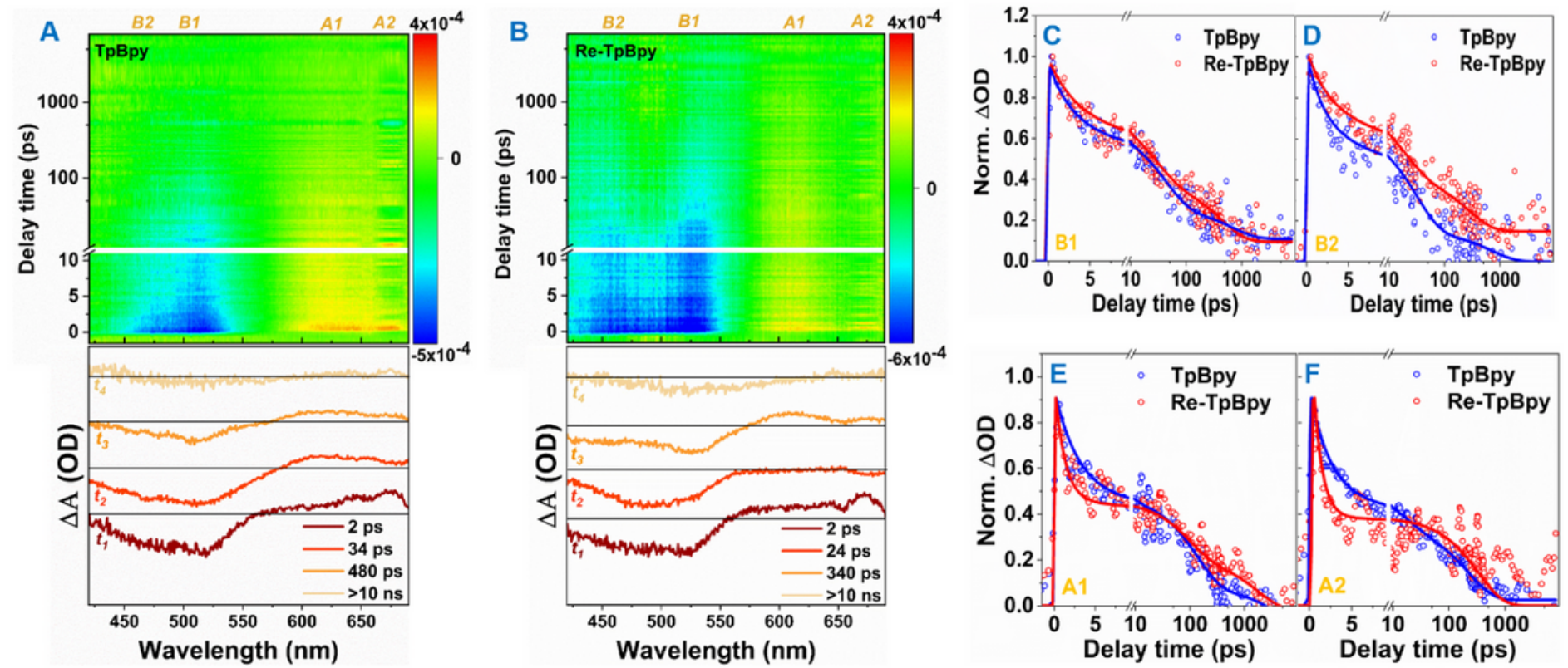

Figure 6 
Two-dimensional transient absorption (TA) spectra under $400 \mathrm{~nm}$ excitation at the fluence of $2 \times 1013$ $\mathrm{ph} / \mathrm{cm} 2$ and the respective SVD fittings of TpBpy (a), Re-TpBpy (b). TA kinetics of two samples at various emission wavelengths representing B1 (c), B2 (d), A1 (e), A2 (f). All spectra are recorded in Nafion (5\% $\mathrm{w} / \mathrm{w}$ in water and 1-propanol).

$530 \mathrm{~nm}$ excitation
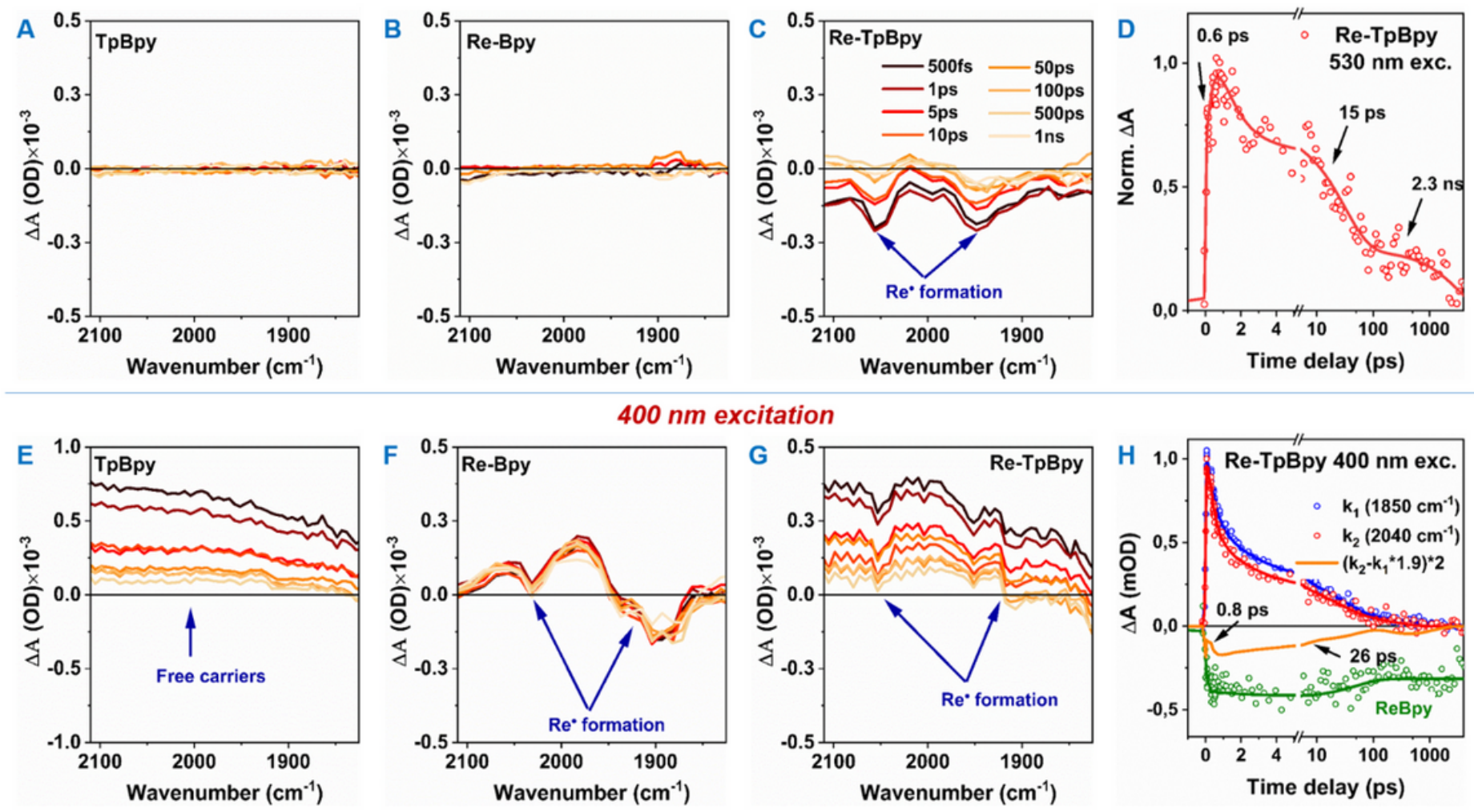

\section{Figure 7}

TRIR spectra of TpBpy, Re-Bpy, and Re-TpBpy excited at $530 \mathrm{~nm}$ (a-c) and $400 \mathrm{~nm}$ (e-g). (d) TRIR kinetics at $2040 \mathrm{~cm}-1$ of Re-TpBpy excited at $530 \mathrm{~nm}$. (h) TRIR kinetics at $2040 \mathrm{~cm}-1$ (red), $1850 \mathrm{~cm}-1$ (blue) and their differential curve (orange) of Re-TpBpy excited at $400 \mathrm{~nm}$. The kinetics at $2040 \mathrm{~cm}-1$ of Re-Bpy excited at $400 \mathrm{~nm}$ is also presented (green). All spectra are recorded in Nafion (5\% w/w in water and 1propanol) 

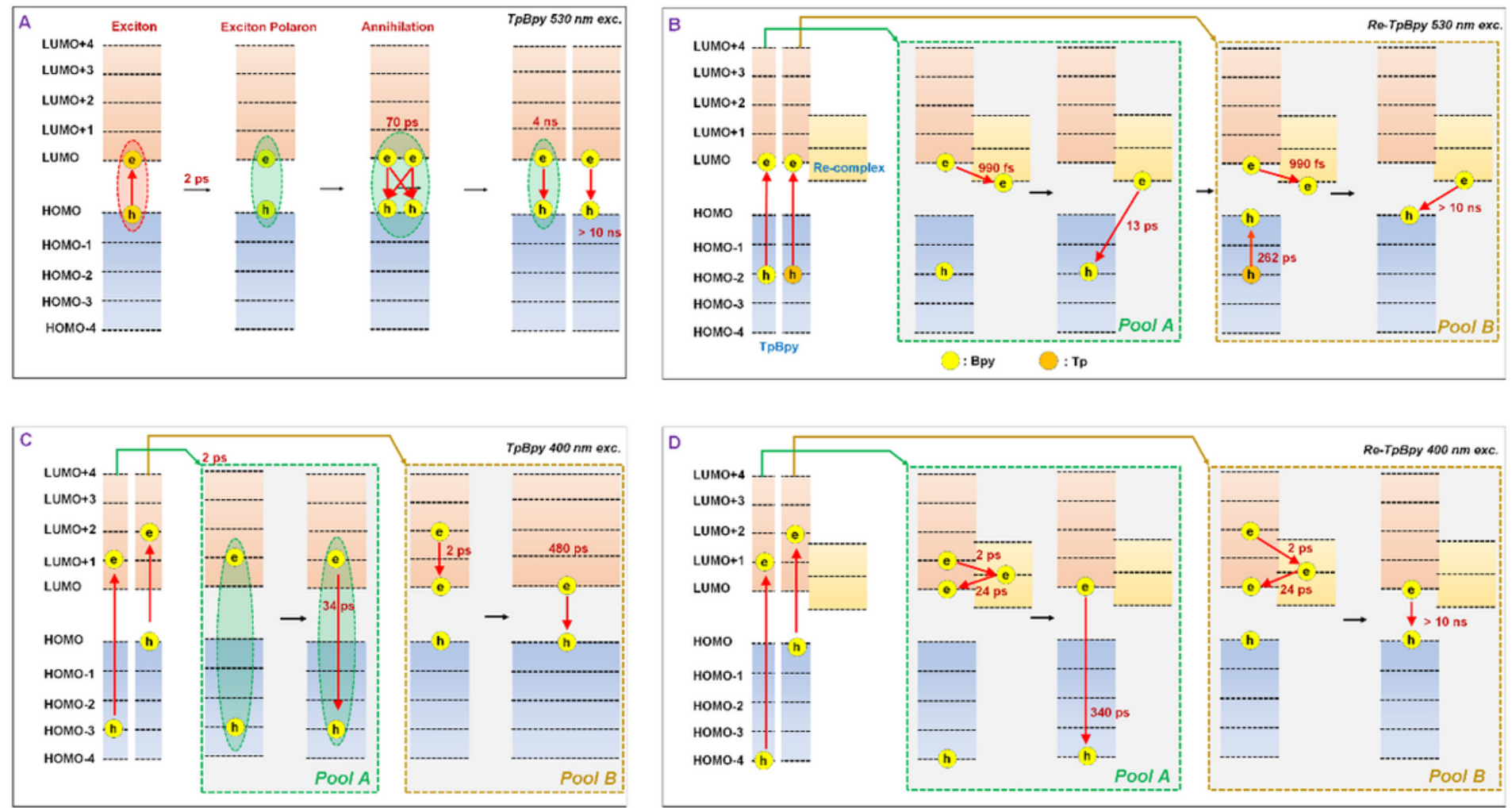

\section{Figure 8}

Schematic diagram of the pathway and lifetime excited state dynamics of TpBpy and Re-TpBpy under a\&b) $530 \mathrm{~nm}$, and c\&d) $400 \mathrm{~nm}$ excitation.
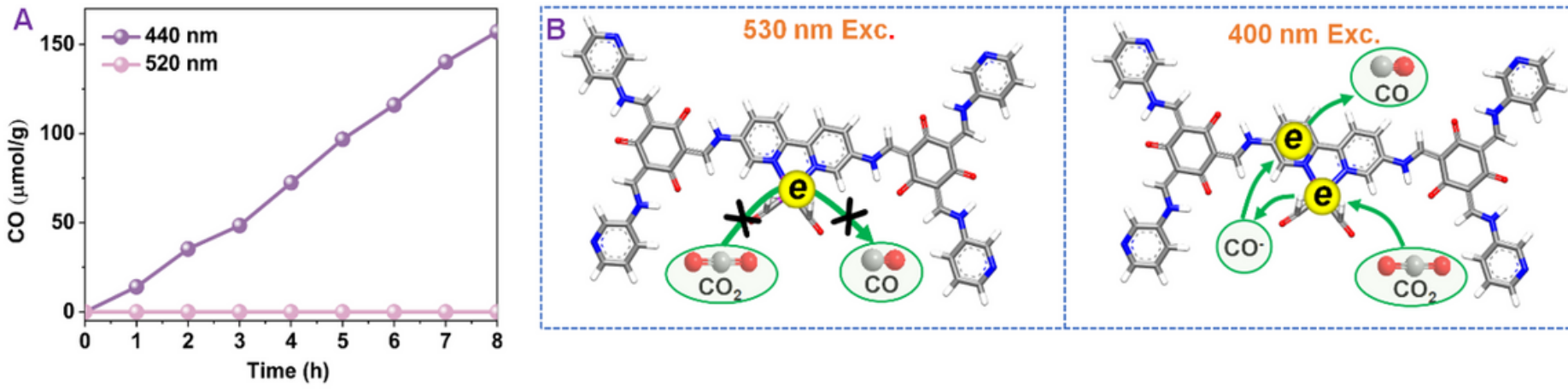

Figure 9

Photocatalytic evolutions of CO by Re-TpBpy under $520 \mathrm{~nm}$ and $440 \mathrm{~nm}$ excitation (a) and Schematic diagram to rationalize the catalytic performance under two excitation conditions (b).

\section{Supplementary Files}

This is a list of supplementary files associated with this preprint. Click to download. 
- Supplementarylnformationfinal.docx

- caculatedUvVisReTpBpynpropanolexc.txt

- caculatedUvVisTpBpynpropanolexc.txt 